\title{
The first technique for non-invasive measurements of volumetric ophthalmic artery blood flow in humans
}

\author{
F Orge, A Harris, L Kagemann, K Kopecky, C W Sheets, E Rechtman, M Zalish
}

Br J Ophthalmol 2002;86:1216-1219

Aim: To validate the first non-invasive measurements of volumetric ophthalmic artery blood flow in humans. Methods: The ophthalmic arteries of healthy normal adults were examined by Advanced Technology Laboratories (ATL, a subsidiary of Phillips Medical Systems Inc) high definition imaging (HDI) 5000 colour Doppler imaging ultrasound with a 5-12 MHZ probe. A group of 14 subjects for experiment 1 and a group of 10 subjects for experiments 2 and 3 were selected, with the examined eye chosen randomly. Peak systolic velocities (PSV) and end diastolic velocities (EDV) of the ophthalmic artery and central retinal artery were measured and recorded. Cineloops (cinegraphic videos) of the ophthalmic arteries were then recorded with the ATL HDI 5000 and values for ophthalmic artery blood flow were produced offline using experimental analysis software. Multiple regression analysis was used to compare blood flow measurements with PSV and EDV measurements in the ophthalmic artery. In two follow up experiments, intraobserver variation in obtaining cineloops and the interanalyser variability in cineloop analysis were studied.

Results: Volumetric flow correlated with ophthalmic artery PSV and EDV ( $\left.p=0.02, r^{2}=0.5\right)$. There was no correlation with the cental retinal artery. The intraobserver coefficient of variation in obtaining cineloops was $29.89 \%$ for blood flow, $19.07 \%$ for diameter, and $22.27 \%$ for velocity. The coefficients of variation of the measurements of the two cineloop analysers were $40.21 \%$ for blood flow, $22.71 \%$ for diameter, and $26.34 \%$ for velocity.

Conclusion: Cineloop analysis produces ophthalmic artery flow measurements which correlate with PSV and EDV, suggesting validity. The intraobserver variation and cineloop analyser variation were found to be in the acceptable range.

U Itrasound technology has advanced on many fronts. The principle of sound waves penetrating a living person, and being scattered by structures within remains the same, but sophisticated advances in the computer analysis of scattered sound waves has introduced three dimensional reconstructions of tissue, and Fourier analysis now provides power Doppler unitless measurements of movement and flow. The purpose of this study is to report a similar new ultrasound analysis technique, and report for the first time ever in vivo volumetric blood flow measurements from the human ophthalmic artery.

These new ultrasound technologies are used to study a variety of vascular disorders. ${ }^{1-4}$ Duplex Doppler ultrasound allows measurement of blood velocity non-invasively, avoiding the use of contrast agents or ionising radiation..$^{5-7}$ Now, calculations of systemic blood flow can be performed based on hundreds of individual arterial diameter and velocity measurements taken over several cardiac cycles. Using this technology, volumetric blood flow has been measured in a number of abdominal, pelvic, and extremity vessels. These include the umbilical vein, ${ }^{8}$ the superior mesentric artery, the lower extremity arteries, such as common femoral artery, ${ }^{10-12}$ superficial femoral artery, popliteal and dorsalis pedis arteries, ${ }^{12}$ and the portal vein. ${ }^{13}$ The objective of our study was the validation of non-invasive measurements of volumetric artery blood flow in the ophthalmic artery (OA) in humans.

\section{METHODS}

\section{Data collection}

All subjects, or their legal guardians, signed an inform consent approved by the university's ethics committee before any measurements were performed. The experiment followed the tenets of the Declaration of Helsinki. In the first experiment the OA of a randomly chosen eye of 14 healthy normal adults (33 (SD 7) years) was examined four times, two times each by two examiners. The subjects had no history of hypertension or diabetes. Systemic blood pressure was measured at the time of each examination, and no person with a systolic pressure greater than $150 \mathrm{~mm} \mathrm{Hg}$ or a diastolic pressure greater than 90 $\mathrm{mm} \mathrm{Hg}$ was included. Potential subjects were excluded for any indication of symptomatic vascular diseases. A history of medication use was also obtained and no volunteer taking prescription drugs that would potentially affect OA blood flow was included.

Ophthalmic arteries were examined by Advanced Technology Laboratories (ATL, a subsidiary of Phillips Medical Systems Inc) high definition imaging (HDI) 5000 colour Doppler imaging (CDI) ultrasound (Bothell, WA, USA) with a L12-5 MHZ linear array transducer. After applying a sterile methylcellulose ultrasound coupling gel to the transducer, it was gently laid over the closed eyelid of the study eye. A plot of blood velocity over time was produced, from which the peak systolic and the end diastolic velocities were measured of both the OA and the central retinal artery (CRA). Pourcelot's resistive index $(\mathrm{RI})^{14}$ was calculated from the measurements automatically by the ATL CDI. Examiners were blinded to their own and each other's measurements.

After completing velocity measurements, the same segment of the OA was located for the cinegraphic recordings (cineloop). A cineloop is a rapid recording of multiple ultrasound frames, sufficient to encompass several cardiac cycles, in other words it is a digital video of the pulsating vessel. The probe was moved into a variety of horizontal, vertical, and rotational positions to ensure visibility of the largest possible segment of the OA within a single slice. With CDI power settings optimised for measurement of the OA, the cineloop was recorded and archived on a magneto optical disk.

Abbreviations: CDI, colour Doppler imaging; CRA, central retinal artery; EDV, end diastolic velocities; OA, ophthalmic artery; PSV, peak systolic velocities 
Cineloop analysis was completed off line using experimental flow analysis software (HDI-lab) developed by ATL. Initially used to assess much larger vessels, the software was modified by ATL with our recommendations for measurement of the OA.

\section{Data analysis}

The cineloop is a video where every colour pixel on every image frame is a colour coded velocity measurement. Analysis of each cineloop requires two basic steps. Firstly, the HDI-lab software must be shown the flow axis of the vessel being measured. This is accomplished by the placement and orientation of an "angle indicator" line (seen in white in Fig 1) over the cineloop. Secondly, the software must be supplied with a measurement or "m-mode" line. Blood flow is measured at all points along the m-mode line within the boundaries of the vessel (seen in yellow in Fig 1). With flow direction and measurement location established, HDI-lab calculates the vessel diameter for each frame of the cineloop throughout the cardiac cycles. The boundary of the vessel is assumed to be where detected movement starts and ends along the m-mode line, in other words the boundary is where the grey scale pixels and the colour pixels touch. These vessel boundaries and the flow axis are used to extrapolate the location of the vessel walls, and calculate diameter. Given the diameter, the HDI-lab software assumes flow through a vessel with a circular cross sectional area. The software then uses the many velocity measurements located along the m-mode line to calculate the volumetric flow for every frame of the cineloop. Since each pixel within the image of the moving blood contains a single velocity measurement, there is no need for assumptions about the turbulence of flow within the vessel. The actual velocity profile across the vessel is presented in each frame of the cineloop. In this particular setup the mean size of a pixel was $0.05 \mathrm{~mm}$. Although with the HDI-lab software the pixel value is affected by the rendering size during quantification, the parameters were kept stable to avoid any shifts.

All cineloop analysis in the following three experiments was performed using identical techniques. Firstly, the angle correction line was positioned parallel to the blood flow vector, and the m-mode line was positioned at 90 degrees to the angle line (Fig l) by using a plastic overlay on the computer screen. Five consecutive measurements, each adjacent to the next, were obtained in this configuration. All five of the velocity, diameter, and flow measurements were recorded, then the average of the five measurements was used for analysis.

In experiment 1 , the data sets from the 14 normal subjects were analysed in order to quantify interexaminer and intraexaminer coefficients of variation of the velocity measurements between the two CDI operators. Cineloop analysis of the OA was correlated with peak systolic velocities (PSV), end diastolic velocities (EDV) of both the OA and the central retinal artery (CRA).

Experiment 2 was designed to calculate the intraobserver variation in obtaining cineloops. For this purpose 10 normal subjects were chosen to be analysed with the same criteria as mentioned above in experiment 1. A single ultrasonographer obtained six cineloops from each subject which were then processed by a single analyser. The cineloop results for each subject were then grouped two by two and compared. The sets were paired as first measurement was compared with the second, the third with the fourth, and fifth with the sixth. So three sets of comparisons were obtained from each of the 10 subjects. Finally, all 30 sets of comparisons were analysed to calculate the coefficient of variation.

Experiment 3 quantified the variability within the software cineloop analysis between two different analysts. Two examiners analysed a cineloop data set, consisting of 26 examinations of normal subjects, each independently using identical analysis techniques.

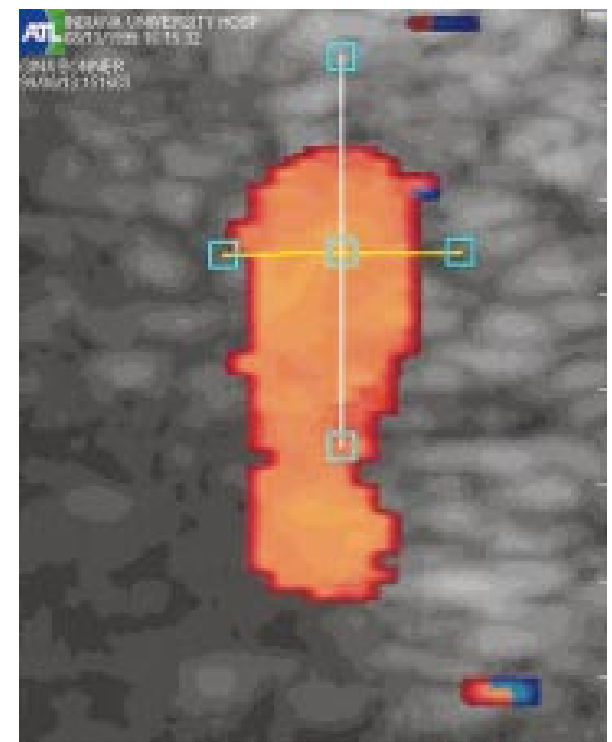

Figure 1 Determination of volumetric blood flow from cineloops requires the analyst to identify the direction of blood flow (white line) and a cross sectional measurement location (yellow line).

\section{RESULTS}

\section{Experiment 1}

Volumetric OA blood flow significantly correlated with PSV (p $\left.=0.0164, r^{2}=0.49\right)$ and EDV $\left(p=0.006, r^{2}=0.587\right)$. Ophthalmic artery PSV and EDV were significantly correlating with flow by multiple regression analysis $\left(\mathrm{p}=0.0231, r^{2}=\right.$ 0.61 ). The volumetric OA blood flow results did not correlate with the CRA readings. The mean OA diameter was 2.02 (SD $0.46) \mathrm{mm}$ (with a range of $1.2-2.5 \mathrm{~mm}$ ), the mean volumetric blood flow was $9.74(3.91) \mathrm{ml} / \mathrm{min}$, and the mean velocity 5.10 ( 1.34$) \mathrm{cm} / \mathrm{s}$.

The intraobserver coefficient of variation in OA PSV, EDV, and RI were $15.32 \%, 21.52 \%$, and $5.79 \%$ for observer 1 ; and $15.33 \%, 23.41 \%$, and $7.1 \%$ for observer 2 . The intraobserver coefficients of variation in CRA PSV, EDV, and RI were $9.05 \%$, $21.1 \%$, and $12.6 \%$ for observer 1 ; and $20.74 \%, 25.78 \%$, and $10.65 \%$ for observer 2 .

The interobserver coefficients of variation in OA PSV, EDV, and RI were $42.59 \%, 37.67 \%$, and $10.33 \%$ respectively. The inter-observer coefficients of variation in CRA PSV, EDV, and RI were $23.5 \%, 34.13 \%$, and $13.94 \%$ respectively.

\section{Experiment 2}

The intraobserver coefficient of variation in obtaining cineloops was $29.89 \%$ for blood flow, $19.07 \%$ for diameter, and $22.27 \%$ for velocity.

\section{Experiment 3}

The coefficient of variation of the measurements of the two cineloop analysers were $40.21 \%$ for blood flow, $22.71 \%$ for diameter, and $26.34 \%$ for velocity.

\section{DISCUSSION}

The new software used in this study is unique for retrobulbar measurements because it uses velocity measurements for every pixel across the width of the vessel to construct a three dimensional map of velocities at a single cross section of the artery. These velocities are collected frame by frame through the duration of several cardiac cycles which enable the software to calculate flow of the ophthalmic artery, both selectively and non-invasively.

Colour Doppler imaging is a highly subjective measurement of retrobulbar haemodynamics. Reproducibility of the velocity measurements in the present study are similar to previously 
established values for the ophthalmic artery. ${ }^{15}$ This suggests that the large variability in the volumetric flow measurements is not due to inexperience in the CDI operators. The high level of variability between the two operators, however, accentuates the importance of utilising a single CDI operator in studies involving multiple measurements. There are three potential sources of noise in the flow data as presented: (1) the acquisition of cineloop data differs slightly from the acquisition of velocity measurements. Reproducibility may improve as the operators gain experience with this new caveat in CDI technology. Operators had 2 years of solid experience and over 4 years of experience in similar techniques. As in almost every imaging technique, the human factor does play a large part, especially when using a new machine in applying a new technique. The obtaining of cineloops is a new entity, and to eliminate the human factors in this technique as much as possible, we believe that further experience would be of great help. (2) Cineloop data may, by their very nature, produce noisier data than velocity measurements; and (3) velocity data are immediately available from the CDI machine, while flow data require offline analysis of cineloops, which includes significant analyst input and interaction.

Measurement of velocity must be performed in the centre of the vessel, which can be located in the centre of any slice through the vessel. The centre of the vessel is initially estimated simply with observation and then after the angle indicator and m-mode lines are appropriately placed. The two lines were moved together in the immediate area until the highest value for flow was achieved. This point of the highest blood flow-diameter values was then referred to as the centre. After setting the angle indicator line, the m-mode line, and finding the centre, flow measurements were taken in the immediate area. It was found that flow values taken along the m-mode line are similar, and values taken at locations slightly off the m-mode line were minimally changed.

Acquisition of cineloop data requires the CDI operator to visualise the longest possible segment of the ophthalmic artery. The ophthalmic artery is a tortuous vessel with numerous branches. Both bends and branch points will introduce turbulence into flow within the ophthalmic artery, creating difficulties in assuming the level of laminar or turbulent flow within. Further, accurate measurement of vessel diameter requires a section of ophthalmic artery with few or no bends. In the present study, the CDI operators used probe rotation and translation until several millimetres of ophthalmic artery, appearing as three to four vessel diameters, free of visible bends and branches, were obtained. This ensures accurate measurement of vessel diameter, as well as providing a sufficiently large area of velocity measurements for placement of the m-mode line. The probe is usually rotated by approximately 15 degrees and swept superiorly and inferiorly across the globe. The exact positioning varies according to the unique vasculature of each individual subject. It is possible that the probe orientation contributed to the variability observed in this study.

In the case of a 7.5 MHZ ultrasound probe, the best possible axial resolution would be a single wavelength, or $0.205 \mathrm{~mm}$. With an ophthalmic artery diameter of approximately $2 \mathrm{~mm}$, the image would consist approximately 10 pixels. A reduction of a single pixel in the measurement would require a decrease of $10 \%$ in vessel diameter. The Doppler shifts that are produced by the blood within the ophthalmic artery, however, are well within the CDI's sensitivity to motion. Higher variability in the diameter measurement, compared to the velocity measurement, was expected.

Acquisition of the cineloop requires recording the entire CDI image frame at a rapid rate, whereas velocity measurements are obtained from a small area contained within the sample window placed by the user, often less than $2 \times 2 \mathrm{~mm}$ in size. Velocity recording from this small area occurs in real time in a continuous manner, and is usually completed in 3-5 seconds. Owing to the large amount of data being generated, cineloop recording occurs at a slower rate, and requires more recording time. The need for an absolute motion-free probe during recording combined with the prolonged recording time for cineloops may also have contributed to the higher variability of the flow data compared to the velocity.

The final source of variability in the generation of volumetric flow measurements is the offline analysis process. In order to examine this source of variability, a single data set was analysed by two analysts using an agreed upon identical technique. Two analysts performing the data analysis of a single data set produced slightly more variability (39.92\%) than the intraobserver variability $(39.55 \%)$ when a single analyst processed both sets of cineloops. It appears that the offline analysis of cineloops may produce the largest source of variability in the quantification of volumetric ophthalmic artery blood flow.

The two components of the blood flow calculation are velocity and diameter. The single data set analysed by two analysts included variability measurements of diameter and velocity as well as flow. The variability of the diameter measurement was greater than that of the velocity measurement using this technique

In vitro models of CDI suggest that parallel changes in PSV and EDV signify changes in volumetric flow. Despite high variability in this experimental technique, a correlation of PSV and EDV with volumetric flow was observed. Despite its highly variability, the relation between PSV, EDV, and flow suggest that the measurement of volumetric flow is valid.

Improvement of the reproducibility of the analysis software appears to offer the most promise in improving the reproducibility of volumetric ophthalmic artery flow measurements.

In a previous study on volumetric flow of the ophthalmic artery published by Michelson and Schuierer, ${ }^{16}$ the inner diameter was estimated with digitalised subtraction angiography of the OA and the mean blood velocity was measured by pulsed Doppler sonography. The inner diameter of the OA was on average $1.14(0.26) \mathrm{mm}$; the mean blood velocity was on average $10.38(4.16) \mathrm{cm} / \mathrm{s}$, and total blood flow in the OA was on average $6.75(3.49) \mathrm{ml} / \mathrm{min}$ per eye. In our study the findings were diameter $2.02(0.46) \mathrm{mm}$, velocity 5.10 (1.34) $\mathrm{cm} / \mathrm{s}$, flow $9.74(3.91) \mathrm{ml} / \mathrm{min}$. There is a significant difference between the findings of the studies but this may be due to subject population. The mean age population in the former study was 58.6 (15.3) years where as in our study the mean age was 33 (7) years. Also in the former study the subjects were neurological patients where as in our study the subjects were healthy adults taking no medications. As the different medical conditions as well as medications can alter the ocular blood flow, the comparison should be made with similar age matched groups and similar health patterns for further conclusions. Other than the studies mentioned above, there are no absolute non-invasive in vivo OA blood flow findings in the literature. It is now possible to obtain direct measurements of volumetric OA blood flow using a single measurement technique. Validation of the ability of cineloop technology to assess accurately, known levels of blood flow in vessels as small as the OA are currently under way.

\section{ACKNOWLEDGEMENT}

Support was provided by an unrestricted grant from Research to Prevent Blindness.

\section{Authors' affiliations}

F Orge, A Harris, L Kagemann, C W Sheets, E Rechtman,

Departments of Ophthalmology and Physiology, Indiana University School of Medicine; Indianapolis, IN, USA

M Zalish, Department of Ophthalmology, Kaplan Medical Center, Rehovot, affiliated with School of Medicine, Hebrew University, Jerusalem, Israel 
K Kopecky, Department of Radiology, Indiana University School of Medicine; Indianapolis, IN USA

Correspondence to: Alon Harris, Department of Ophthalmology, Indiana University School of Medicine, Indianapolis, IN, USA:

alharris@indiana.edu

Accepted for publication 29 May 2002

\section{REFERENCES}

1 Satumora S. Study of flow patterns in peripheral arteries by ultrasonics. J Acoust Soc Jpn 1959;15:151-4.

2 Baker DW. Pulsed ultrasonic Doppler flow sensing. IEEE Trans Sonics Ultrasonics 1970;17:170-85.

3 Hokanson DE, Mozersky DJ, Summer DS, et al. Ultrasonic arteriography: a new approach to arterial visualization. Biomed Eng $1971 ; 6: 420$

4 Jäger KA, Ricketts HJ, Strandness DE. Duplex scanning for the evaluation of lower limb arterial disease. In: Bernstein EF, ed. Noninvasive diagnostic techniques in vascular disease. Princeton, NJ: CV Mosby, 1985:619-31.

5 Burns PN, Jaffe CC. Quantitative flow measurements with Doppler ultrasound: Techniques, accuracy, and limitations. Radiol Clin North Am 1985;23:641-57.

6 Taylor KJW, Holland SK. Doppler US: Part I. Basic principles, instrumentation, and piffalls. Radiology 1990;174:297-307.
7 Holland CK, Taylor KJW. Blood flow quantification: waveform analysis, volume measurement, tumor flow and the role of color imaging. In: Wells PNT, ed. Advances in ultrasound techniques and instrumentation. New York: Churchill Livingstone, 1993:125-39.

8 Gill RW, Kossoff G, Warren PS, et al. Umbilical venous flow in normal and complicated pregnancy. Ultrasound Med Biol 1984;10:349-63.

9 Qamar MI, Read AE, Skidmore R, et al. Transcutaneous Doppler ultrasound measurement of superior mesenteric artery blood flow in man. Gut 1986;27:100-5.

10 Lewis P, Psalia JV, Davies WT, et al. Measurement of volume flow in the human common femoral artery using a duplex ultrasound system. Ultrasound Med Biol 1986;12:777-84.

11 Field JP, Musson AM, Zwolak RM, et al. Duplex arterial flow measurements in normal lower extremities. J Vasc Tech 1989;13:13-19.

12 Holland CK, Brown JM, Scoutt LM, et al. Lower extremity volumetric arterial blood flow in normal subjects. Ultrasound Med Biol 1998:24:1079-86.

13 Moriyasu F, Ban N, Nishida O, et al. Clinical application of an ultrasonic duplex system in the quantitative measurement of portal blood flow. J Chin Ultrasound 1986;14:579-88.

14 Pourcelot L. Applications cliniques de l'examen Doppler transcutane. In: Peronneau $\mathrm{P}$, ed. Velocimetrie ultrasonore Doppler. INSERM 1974;34:213-40

15 Williamson T.H., Harris, A. Color Doppler ultrasound imaging of the eye and orbit. Surv Ophthalmol 1996;40:255-67.

16 Michelson G, Schuierer G. [Absolute blood flow in the opthalmic artery.] Fortschr Ophthalmol 1991;88:687-9.

\section{New BJO online submission and review system}

The Editors of British Journal of Opthalmology are pleased to inform authors and reviewers of its new online submission and review system. Bench $>$ Press is a fully integrated electronic system which uses the internet to allow rapid and efficient submission of manuscripts, as well as the entire peer review process to be conducted online.

Authors can submit their manuscript in any standard word processing software. Graphic formats acceptable are: .jpg, .tiff, .gif, and eps. (Nb. Multipage PowerPoint presentations are NOT acceptable.) Text and graphic files are automatically converted to PDF for ease of distribution and reviewing purposes. Authors are asked to approve their submission before it formally enters the reviewing process.

To access the system click on "SUBMIT YOUR MANUSCRIPT HERE" on the BJO homepage: http://www.bjopthalmol.com/, or you can access Bench>Press directly at http://submit-bjo.bmijournals.com/.

We are very excited with this new development, it really is simple to use and should be a big improvement on the current peer review process. Full instructions can be found on Bench>Press and BJO online. Please contact Natalie Davies, Project Manager, ndavies@bmigroup.com for further information.

\section{Pre-register}

We would be grateful if all British Journal of Opthalmology authors and reviewers pre-registered with the system. This will give you the opportunity to update your contact and expertise data, allowing us to provide you with a more efficient service.

\section{Instructions For Registering}

1. Enter http://submit-bjo.bmijournals.com

2. Click on "Create a New Account" in the upper left hand side of the Bench>Press

homepage.

3. Enter your email address in the space provided.

4. Choose a password for yourself and enter it in the spaces provided.

5. Complete the question of your choice to be used in the event you cannot remember your password at a later time.

6. Click on the "Save" button at the bottom of the screen.

7. Check the email account you registered under. An email will be sent to you with a verification number and URL.

8. Once you receive this verification number, click on the URL hyperlink and enter the verification number in the relevant field. This is for security reasons and to check that your account is not being used fraudulently.

9. Enter/amend your contact information, and update your expertise data.

10. Please note: You only need to create a new account once. If you submit to another

BM Publishing Group journal you can use the same email address and password 